\title{
Hand Hygiene Practices among Nursing Staff in Public Secondary Care Hospitals in Kuwait: Self-Report and Direct Observation
}

\author{
Batool Al-Wazzan ${ }^{a}$ Yasmeen Salmeen ${ }^{a}$ Eisa Al-Amiri ${ }^{a} \quad$ Ala'a Abul $^{a}$ \\ Manal Bouhaimed ${ }^{a, b} \quad$ Abdullah Al-Taiar ${ }^{a}$ \\ Departments of a Community Medicine and Behavioural Science and ${ }^{b}$ Surgery, Faculty of Medicine, \\ Kuwait University, Jabriya, Kuwait
}

\section{Key Words}

Hand hygiene $\cdot$ Nurses $\cdot$ Hospital $\cdot$ Kuwait $\cdot$ Gulf $\cdot$ Middle East

\begin{abstract}
Objective: To assess the compliance with hand hygiene guidelines among nursing staff in secondary care hospitals in Kuwait. Subjects and Methods: A cross-sectional study was conducted through direct observation using the Lewisham observation tool and self-administered questionnaire in six major public secondary care hospitals in Kuwait. Only patient care activities that are described as 'dirty contacts' by the Fulkerson scale were considered as indications for hand hygiene while any attempt for hand hygiene was considered as compliance. A self-administered questionnaire was prepared and pilot tested and then distributed to nursing staff at each ward immediately after conducting the inspection; 550 were distributed and 454 were completed and returned. Among 204 observation sessions, a total of 935 opportunities and 312 hand hygiene practices were recorded. Results: The overall compliance was $33.4 \%$. The observed compliance significantly varied between different ward categories from $14.7 \%$ in emergency to $55 \%$ in medical wards. Of the 454 nursing staff who participated in self-reported compliance, 409 (90\%) indicated that they always washed their hands upon practicing patient care activities. Nurses consis-
\end{abstract}

tently reported higher compliance after conducting patient care activities rather than before. Being busy with work (42.2\%), having sore/dry hands (30.4\%) and wearing gloves (20.3\%) were the most frequently reported hindrances to improving hand hygiene. Conclusion: Observed hand hygiene compliance among nursing staff in secondary care hospitals in Kuwait was poor. High self-reported compliance may reflect a high level of awareness of hand hygiene but may also suggest that improving compliance through increasing awareness has probably reached saturation.

Copyright $\odot 2011$ S. Karger AG, Basel

\section{Introduction}

Currently, hospital-acquired infections are recognized as a major challenge and complicate $4-10 \%$ of hospital admissions [1-3]. It is estimated that 1 in 10 hospitalized patients acquires an infection after admission [4], resulting in 80,000 deaths in North America alone [2]. Hospital-acquired infections result in longer hospital stays and additional investigations and treatments, adding considerably to the cost of delivering health services, and are an economic burden on society.

It has been shown that hand hygiene (either as hand washing or hand disinfection) is the single most important preventive measure against hospital-acquired infec-

\section{KARGER}

Fax +4161306 1234

E-Mail karger@karger.ch

www.karger.com
(C) 2011 S. Karger AG, Basel

1011-7571/11/0204-0326\$38.00/0

Accessible online at:

www.karger.com/mpp
Dr. Abdullah Al-Taiar

Department of Community Medicine and Behavioural Science

Faculty of Medicine, Kuwait University, P.O. Box 24923

Safat 13110 (Kuwait)

Tel. +965 2498 6553, E-Mail altaiar@ hsc.edu.kw 
tions [5-7]. However, the importance of this procedure is not well recognized by health care providers [8], resulting in poor compliance. Most studies that have investigated compliance with hand hygiene among healthcare workers generally reported poor compliance $[9,10]$.

Measuring the compliance with hand hygiene is not an easy task, with direct observation being described as the gold standard although the reliability of the tools used in direct observation remains questionable [11]. Recently, attempts have been made to improve the reliability and the validity of the tools used to audit hand hygiene behaviour in health services [12]. Currently, direct observation is used to monitor hand hygiene and provide performance feedback to improve hand hygiene among healthcare workers.

This study aimed to investigate the compliance of nursing staff in secondary care hospitals in Kuwait. Nursing staff in secondary care hospitals in Kuwait come from different countries and display considerable diversity in cultural beliefs and prior training, factors that can influence hand hygiene $[13,14]$. Our aim was to assess the compliance with hand hygiene guidelines among nursing staff in public secondary care hospitals through direct observation and self-reporting and to explore the perceived factors that hinder compliance.

\section{Subjects and Methods}

\section{Study Hospitals and Study Participants}

Public health services in Kuwait provide $80 \%$ of healthcare services under three categories: primary, secondary and tertiary. There are six health regions; each has a general secondary level hospital and referring primary healthcare centres.

The study was conducted in 2009 at all six public secondary care hospitals, namely Amiri, Mubarak, Sabah, Farwaniya, Adan and Jahra. It covered 84 wards under five ward categories: Pediatrics, Surgery, Medicine, Intensive Care Units/Critical Care Units and Emergency. The hospitals have similar structural building designs and facilities. In the general patients' rooms there are 2-4 beds which are extended to 6 in busy hospitals. The number of beds in each ward varies from 137 (range 112-183) in medical wards to 107 (range 64-144) in surgical wards and 95 (range 56-163) in pediatric wards. Two sinks in each room are usually available for patients' use. In each ward there is a treatment room where nurses prepare medications and conduct some patient care activities. In the treatment room there are 1 or 2 washing basins with antiseptic soap and disposable paper-based hand towels. Alcohol-based hand rub dispensers are either wall mounted or free standing on trolleys or tables and distributed in patient care areas. Intensive care units usually have better facilities for hand washing compared with other ward categories.

Nursing staff in Kuwait are mostly foreigners with a huge diversity in their culture and training. Out of 12,188 nurses working in Kuwait in 2009, only 804 (6.6\%) were Kuwaiti nationals [15]. In this study, nursing staff were defined as any nurse who provides care for patients regardless of her/his professional rank.

The study was approved by the Ethics Committee, Health Sciences Centre, Kuwait University and the Ministry of Health, State of Kuwait.

\section{Study Design and Data Collection}

A cross-sectional study was conducted using two different approaches to assess hand hygiene practices among nursing staff. First, we performed direct observations using the Lewisham observational tool [16] with at least 2 sessions of 20 min each in every ward. The Lewisham tool, which has been used in monitoring adherence to hand hygiene, is based on the principle that when doing a procedure (e.g. taking a blood sample), the nurse has a 'hand hygiene opportunity' (O). When a nurse washes his/her hands, the nurse has a 'hand hygiene practice' $(\mathrm{H})$. An observer records the care activities performed $(\mathrm{O})$ and hand hygiene performance $(\mathrm{H})$ by any nurse in a 20 -min period. Compliance is calculated as a percentage, i.e. compliance $\%=$ observed hand hygiene $(\mathrm{H}) \div$ hand hygiene opportunity $(\mathrm{O}) \times 100$.

A list of opportunities guided by the Infection Control Policy in Kuwait was prepared. The policy identified three hand-washing categories classified according to type, intensity and duration of activity. The categories were social hand washing, hygienic hand washing (also termed 'hand antisepsis'), and surgical hand washing (scrubbing). Only patients' contacts and patient care activities that are listed as indications for hygienic hand washing or surgical hand washing were identified as opportunities for hand hygiene. These patient care activities are categorized as 'dirty contacts' according to the scale devised by Fulkerson [17].

Hand hygiene practice $(\mathrm{H})$ was defined as any attempt to clean hands with water alone, with water and any hand-washing products, or with alcohol-based hand rubs. No judgment was made on the duration, the efficacy, the quality or the appropriateness of hand hygiene practices. Data on potential factors that influence observed compliance with hand hygiene, including ward category (e.g. surgery, medicine), type of shift (morning, evening, night) and patient-to-nurse ratio at the time when the observations were collected.

Four senior medical students (B.W., Y.S., E.A., A.A.) recorded potential opportunities for and actual performance of hand hygiene in observation sessions distributed in different working shifts (morning, evening and night shifts). Each item in the observation list was discussed to reach a consensus on the way the observational tool should be used. Data were recorded on a specially designed form that was pilot tested in five sessions. Data were collected by two observers at the same time in a few sessions to calculate inter-observer agreement $(\kappa=72.2 \%)$. The observers followed a standardized operating procedure from introducing themselves to the head nurse at the departmental level until they finished data collection in the ward.

It has been suggested that the behavior of an individual or a group will change to meet the expectations of the observer if they are aware their behavior is being observed (Hawthorne effect). In order to minimize the Hawthorne effect, observers did not provide details of the study procedures. The observer found a place where he/she could watch both the contacts or patient care activities and hand hygiene practices but causing minimum or no obstruction of work in the ward. 
Table 1. Observed hand hygiene compliance among nursing staff in public secondary care hospitals in Kuwait

\begin{tabular}{lclc}
\hline Hospital & $\begin{array}{l}\text { Oppor- } \\
\text { tunities }\end{array}$ & $\begin{array}{l}\text { Observed } \\
\text { compliance, } \\
\mathrm{n}(\%)\end{array}$ & $\begin{array}{l}\text { Self-reported } \\
\text { compliance, } \\
\mathrm{n}(\%)\end{array}$ \\
\hline Al-Amiri & 166 & $72(43.4)$ & $41(74.6)$ \\
Mubarak Al-Kabeer & 204 & $72(35.3)$ & $56(67.5)$ \\
Al-Adan & 175 & $61(34.8)$ & $50(80.6)$ \\
Al-Sabah & 132 & $44(33.3)$ & $69(71.9)$ \\
Farwaniya & 139 & $42(30.2)$ & $63(70.8)$ \\
Jahra & 119 & $21(17.6)$ & $54(81.8)$ \\
Total & 935 & $312(33.4)$ & $333(73.8)$ \\
\hline Ward & & & $90(70.9)$ \\
Medicine & 151 & $84(55.6)$ & $92(85.2)$ \\
ICU/CCU & 146 & $66(45.2)$ & $78(86.7)$ \\
Pediatric & 136 & $59(43.4)$ & $85(57.0)$ \\
Surgery & 115 & $46(40.0)$ & $8(66.7)$ \\
Emergency & 387 & $57(14.7)$ & $333(73.8)$ \\
Total & 935 & $312(33.4)$ & \\
\hline
\end{tabular}

Table 2. Socio-demographic characteristics of 454 nursing staff in public secondary care hospitals in Kuwait

\begin{tabular}{lcc}
\hline Variable & Number & Percent \\
\hline Gender $^{1}$ & & \\
$\quad$ Female & 376 & 83.0 \\
Marital status & & \\
$\quad$ Married & & 79.2 \\
Single & 358 & 20.8 \\
Nationality & 94 & \\
Indian & & 64.8 \\
Filipino & 294 & 12.3 \\
Egyptian & 56 & 6.4 \\
Kuwaiti & 29 & 4.8 \\
Indonesian & 22 & 3.7 \\
Palestinian/Jordanian & 17 & 1.8 \\
Others & 8 & 6.2 \\
\hline
\end{tabular}

\footnotetext{
${ }^{1}$ Missing for one participant.

2 Two participants were widowed or divorced.
}

After completion of direct observation, self-assessment of compliance was conducted in the same ward but not necessarily by the same observed nurses. We used a simplified self-administered questionnaire that included questions assessing the frequency of hand washing according to five options (always, often, sometimes, never, not applicable). A specific list of patient care activities was established, guided by the Infection Control Policy, such as fixing an intravenous or a urinary catheter. Self-reported compliance was defined as the percentage of 'always' responses for each listed care activity excluding those who have never done that care activity (their response was 'not applicable'). The questionnaire was developed in English and then translated into Arabic and pilot tested. The English and Arabic versions were available for the nurses in wards.

Data Analysis

Data were entered and analyzed using Statistical Package for Social Sciences (SPSS) version 16 . The $\chi^{2}$ test was used to test for the difference in proportions and Spearman correlation was used to investigate the association between compliance and patient-tonurse ratio.

\section{Results}

\section{Direct Observation}

Data were collected from 204 observation sessions (20-minutes each) in a 1-week period, providing $68 \mathrm{~h}$ of observation time. A total of 935 opportunities and 312 hand hygiene practices were observed.

The overall compliance was $33.4 \%$ and varied significantly between hospitals $(\mathrm{p}=0.04)$ (table 1$)$. The highest observed compliance was in Amiri Hospital: $43.4 \%$ and the lowest in Jahra Hospital: 17.6\%. Also, there was a significant difference in the observed compliance between different ward categories $(\mathrm{p}<0.001)$ (table 1$)$. The highest compliance was observed in medical wards: $55.6 \%$ and the lowest in the emergency wards: $14.7 \%$. The observed compliance in emergency wards in the morning, evening and night shifts was 11.1, 13.6 and $19.7 \%$, respectively.

Except for the emergency wards, in which the number of patients changed continuously, the patient-to-nurse ratio was noted during each observation session. There was no correlation between the patient-to-nurse ratio and the observed compliance (Spearman's $\rho=-0.04, \mathrm{p}=0.70$ ).

\section{Self-Assessment Compliance}

In self-reported compliance, of the 550 questionnaires distributed 454 were completed and returned, thereby yielding a response rate of $82.5 \%$. The mean \pm SD age of the study group was $33 \pm 7.1$ years; $83 \%$ were females. Other socio-demographic characteristics of the study group are given in table 2. More than half of the nurses came from India while Kuwaitis represented approximately $5 \%$ of the study group. Self-reported compliance using specific examples of patient care activities from the Infection Control Policy is given in table 3. More than $90 \%$ of the participants reported that they always washed their hands when engaging in activities that require hy- 
gienic hand washing as described by the Infection Control Policy. These included washing their hands before fixing a urinary catheter, feeding a neonate or changing a wound dressing. However, only 86.2 and $82.0 \%$ reported washing their hands before fixing an intravenous catheter or taking a blood sample, respectively. There was no significant variation in self-reported compliance between hospitals with respect to any patient care activity. However, significant differences in self-reported compliance were found between different wards, with nurses in intensive/critical care units and pediatric wards consistently reporting higher compliance.

Interestingly, more than $91 \%$ of the nurses reported always washing their hands after all care activities. For example, practically all participants reported that they always wash their hands after changing wound dressing or dealing with a urine sample (table 3 ). Moreover, a higher proportion reported washing their hands after their shift in comparison with the start of their shift (96.9 and $59.3 \%$, respectively, $\mathrm{p}<0.001)$.

With respect to knowledge and awareness of infection control guidelines, 431 (95\%) were aware of the existence of guidelines. More than $97 \%$ of nurses self-rated their knowledge about hand hygiene and their hand hygiene practices as excellent or good although more than $83 \%$ perceived room for improvement in their hand hygiene practices (table 3 ).

Factors that hinder hand hygiene practices from nurses' perspective are shown in table 4 . Being busy with work and having sore or dry hands were the most frequently reported factors, 185 (42.2\%) and 130 (30.4\%), respectively. Other frequently mentioned factors included not enough disposable paper-based hand towels and inappropriate location of the sink.

\section{Discussion}

The direct observations showed that compliance with hand hygiene among nursing staff working at secondary care hospitals in Kuwait is poor (33\%). This level of hand hygiene compliance is less than that reported among nursing staff in most European settings [18]; however, it is higher than that reported in Saudi Arabia [10], Spain [19] or Italy [20]. While 33\% compliance is low, it is likely that the true compliance could be even lower, most probably because of the strict definition of hand hygiene opportunities (only dirty contacts according to the scale devised by Fulkerson) and the inclusive definition for hand hygiene practices (any attempt to practice hand hygiene).
Table 3. Self-reported compliance with hand-washing guidelines among 454 nursing staff in public secondary care hospitals in Kuwait $^{1}$

\begin{tabular}{|c|c|c|}
\hline & $\begin{array}{l}\text { Number/ } \\
\text { total number }\end{array}$ & Percent \\
\hline \multicolumn{3}{|l|}{ Hand washing before the following activities ${ }^{2}$} \\
\hline Feeding neonate or newborn & $269 / 281$ & 95.7 \\
\hline Changing wound dressing & $416 / 441$ & 93.7 \\
\hline Fixing urinary catheter & $398 / 428$ & 93.0 \\
\hline Fixing intravenous catheter & $388 / 450$ & 86.2 \\
\hline Taking blood sample & $368 / 449$ & 82.0 \\
\hline Wearing gloves & $267 / 435$ & 61.4 \\
\hline \multicolumn{3}{|l|}{ Measuring blood pressure of diabetic/patient } \\
\hline \multicolumn{3}{|l|}{ Hand washing after the following activities ${ }^{2}$} \\
\hline Changing wound dressing & $442 / 443$ & 99.8 \\
\hline Dealing with urine sample & $447 / 449$ & 99.6 \\
\hline Taking blood sample & $435 / 448$ & 96.7 \\
\hline Removing gloves & $431 / 450$ & 95.8 \\
\hline Taking temperature of patient with sepsis & $409 / 446$ & 91.7 \\
\hline \multicolumn{3}{|l|}{ Hand washing before/after work } \\
\hline At the start of the shift ${ }^{3}$ & 262 & 59.3 \\
\hline At the end of the shift ${ }^{4}$ & 431 & 96.9 \\
\hline Upon resuming work after a break ${ }^{5}$ & 374 & 85.0 \\
\hline \multicolumn{3}{|l|}{ Self-rated knowledge on hand hygiene $e^{6}$} \\
\hline Excellent/good & 433 & 97.0 \\
\hline Fair/poor & 13 & 2.9 \\
\hline \multicolumn{3}{|l|}{ Self-rated hand hygiene practices ${ }^{7}$} \\
\hline Excellent/good & 441 & 98.2 \\
\hline Fair & 8 & 1.8 \\
\hline $\begin{array}{l}\text { Perceived room for improvement in hand } \\
\quad \text { hygiene practices }\end{array}$ & 365 & 83.7 \\
\hline \multicolumn{3}{|c|}{$\begin{array}{l}{ }^{1} \text { Number and percentage of nurses who reported that they 'always' } \\
\text { wash their hand before or after care activity. } \\
{ }^{2} \text { People who answered 'not applicable' were excluded, which resulted } \\
\text { in different denominators. } \\
{ }^{3} \text { Missing for } 12 \text { participants. } \\
{ }^{4} \text { Missing for } 9 \text { participants. } \\
{ }^{5} \text { Missing for } 14 \text { participants. } \\
{ }^{6} \text { Missing for } 8 \text { participants. } \\
{ }^{7} \text { Missing for } 5 \text { participants. } \\
{ }^{8} \text { Missing for } 18 \text { participants. }\end{array}$} \\
\hline
\end{tabular}

Table 4. Perceived factors that hinder hand-washing practice as reported by 454 nursing staff in public secondary care hospitals in Kuwait

\begin{tabular}{lc}
\hline Factors that hinder hand washing & Number \\
\hline Too busy with work & $185(42.2)$ \\
Hands become sore or dry & $130(30.4)$ \\
Not enough disposable paper-based hand towels & $100(23.0)$ \\
Gloves worn most of the time & $88(20.3)$ \\
Sink location is not appropriate & $71(16.6)$ \\
Not enough alcohol-based rub & $39(9.0)$ \\
Not enough soap & $38(8.7)$ \\
Others & $6(1.3)$ \\
\hline
\end{tabular}


In addition, the Hawthorne effect should have resulted in improved hand hygiene compliance.

While the compliance by direct observation was low in all six hospitals, it showed significant variation between the hospitals, which may reflect variation in the institutional conditions that encourage safety $[9,13]$. Another source of variation was in the type of patient care unit; the compliance was much lower in the emergency wards in comparison with medical, pediatric or surgical wards. This is consistent with findings from other studies, which have suggested that compliance with hand hygiene worsens when the demand for hand hygiene is high $[21,22]$. Most of the hand hygiene opportunities (41.5\%) in our study occurred in the emergency wards, reflecting a high demand for hand hygiene. Alcohol-based hand rubs have been suggested as a potential solution in highdemand situations like emergency wards in a previous study [9] because they save time [23] and cause less irritation to hands [24]. We therefore suggest that the supply of alcohol-based hand rubs in emergency wards should be carefully monitored to avoid shortage due to high demand.

Non-compliance with hand hygiene among healthcare workers is a complex problem. Several methods have been used to promote compliance among healthcare workers such as providing performance feedback [25,26], engaging patient as an observer for hand hygiene [27] and using an electronic surveillance device [28]. Frequent monitoring of hand hygiene and providing feedback on performance based on behavioural sciences theory should be explored in Kuwait. One point that should be stressed is the diversity of the background of nursing staff in Kuwait, which is likely to complicate interventions that aim to improve compliance.

While compliance by direct observation was low, selfreported compliance was extremely high and reached more than $90 \%$ in most patient care activities that require hygienic hand washing according to the national guidelines. This supports conclusions from other studies that observed practice is unrelated or weakly correlated to self-reported behaviour [22, 29]. While the design of our study does not allow direct comparison between self-reported and observed behaviour on the level of individual nurses, it is obvious that using self-reported behaviour would dramatically overestimate compliance.

In self-reported compliance, nurses consistently reported higher compliance after conducting patient care activities rather than before conducting these activities. Similar findings have been reported from other studies $[19,20,29]$, which has been attributed to the assumption that nurses are more likely to use hand hygiene to protect themselves rather than the patient [20], or to avoid transmitting an infection to consecutive patients rather than to infect the first patient [29].

One in 5 nurses reported wearing gloves as a hindrance to hand washing, which supports the assertion that nurses believe that wearing gloves is a substitute for hand washing or hand hygiene practices [9,21]. It is important to increase the awareness that hand hygiene is required whether or not gloves are used [30].

Approximately $30 \%$ of nurses reported fear of sore or dry hands as a hindrance for hand washing, which is consistent with the findings from other studies $[9,21]$. While it is widely known that frequent hand washing with hygienic soap or antiseptic agents can cause dryness and irritation to the skin, moisturizing alcohol-based hand rubs offer a potential solution [9].

As in other studies that have used direct observations to investigate compliance with hand hygiene, nurses may have changed their behaviour or improve their hand hygiene practices because they were being observed (i.e. the Hawthorne effect). Only the head nurses (at the department level not ward level) were informed that hand hygiene was to be inspected, but they were not informed about the activities that were to be observed. Data collectors were senior medical students who are usually present on the wards and, therefore, their presence was unlikely to provoke a change in behaviour. We used a restrictive definition for hand hygiene opportunities and a permissive definition for hand hygiene compliance, which may have overestimated the compliance.

\section{Conclusion}

The observed compliance with hand hygiene among nursing staff working at secondary care hospitals in $\mathrm{Ku}$ wait was poor. Self-reported compliance was twice to three times the observed compliance, suggesting that self-reported compliance has little value in this setting. Extremely high self-reported compliance may reflect a high level of awareness among nurses but may also indicate that improving compliance through increasing awareness has probably reached saturation. Frequent auditing for hand hygiene and properly applied feedback on performance should be explored to promote hand hygiene practices in hospitals in Kuwait. 


\section{References}

1 Stamm AM, Long MN, Belcher B: Higher overall nosocomial infection rate because of increased attack rate of methicillin-resistant Staphylococcus aureus. Am J Infect Control 1993;21:70-74.

$>2$ Boyce JM: It is time for action: improving hand hygiene in hospitals. Ann Intern Med 1999;130:153-155.

-3 Aly NY, Al-Mousa HH, Al Asar el SM: Nosocomial infections in a medical-surgical intensive care unit. Med Princ Pract 2008;17: 373-377.

$\checkmark 4$ Haley RW: Measuring the costs of nosocomial infections: methods for estimating economic burden on the hospital. Am J Med 1991;91:32S-38S.

$\checkmark 5$ Larson EL: APIC guideline for handwashing and hand antisepsis in health care settings. Am J Infect Control 1995;23:251-269.

$>6$ Pittet D, Hugonnet S, Harbarth S, Mourouga P, Sauvan V, Touveneau S, Perneger TV: Effectiveness of a hospital-wide programme to improve compliance with hand hygiene. Infection Control Programme. Lancet 2000; 356:1307-1312.

7 Pittet D: Improving compliance with hand hygiene in hospitals. Infect Control Hosp Epidemiol 2000;21:381-386.

$>8$ Jarvis WR: Handwashing - the Semmelweis lesson forgotten? Lancet 1994;344:13111312 .

$>9$ Pittet D: Improving adherence to hand hygiene practice: a multidisciplinary approach. Emerg Infect Dis 2001;7:234-240.

-10 Basurrah MM, Madani TA: Handwashing and gloving practice among health care workers in medical and surgical wards in a tertiary care centre in Riyadh, Saudi Arabia. Scand J Infect Dis 2006;38:620-624.

11 Gould DJ, Chudleigh J, Drey NS, Moralejo D: Measuring handwashing performance in health service audits and research studies. J Hosp Infect 2007;66:109-115.
12 McAteer J, Stone S, Fuller C, Charlett A, Cookson B, Slade R, Michie S: Development of an observational measure of healthcare worker hand-hygiene behaviour: the handhygiene observation tool (HHOT). J Hosp Infect 2008;68:222-229.

13 Cantrell D, Shamriz O, Cohen MJ, Stern Z, Block C, Brezis M: Hand hygiene compliance by physicians: marked heterogeneity due to local culture? Am J Infect Control 2009;37: 301-305.

14 Allegranzi B, Memish ZA, Donaldson L, Pittet D: Religion and culture: potential undercurrents influencing hand hygiene promotion in health care. Am J Infect Control 2009;37:28-34

15 Al-Jarallah KF, Moussa MA, Hakeem SK, Al-Khanfar FK: The nursing workforce in Kuwait to the year 2020. Int Nurs Rev 2009; 56:65-72.

16 Webpage: Available from: http://www.cleansafe-care.nhs.uk/ArticleFiles/Files/Lewisham_hand_hygiene_audit_tool_V2.doc. Accessed on 10 Mar 2009.

17 Fox MK, Langner SB, Wells RW: How good are hand washing practices? Am J Nurs 1974; 74:1676-1678.

18 Moret L, Tequi B, Lombrail P: Should selfassessment methods be used to measure compliance with handwashing recommendations? A study carried out in a French university hospital. Am J Infect Control 2004; 32:384-390.

19 Novoa AM, Pi-Sunyer T, Sala M, Molins E, Castells X: Evaluation of hand hygiene adherence in a tertiary hospital. Am J Infect Control 2007;35:676-683.

20 Pan A, Domenighini F, Signorini L, Assini R, Catenazzi P, Lorenzotti S, Patroni A, Carosi G, Guerrini G: Adherence to hand hygiene in an Italian long-term care facility. Am J Infect Control 2008;36:495-497.

21 Pittet D: Compliance with hand disinfection and its impact on hospital-acquired infections. J Hosp Infect 2001;48 Suppl A:S40-46.
22 O’Boyle CA, Henly SJ, Larson E: Understanding adherence to hand hygiene recommendations: the theory of planned behavior. Am J Infect Control 2001;29:352-360.

23 Voss A, Widmer A: No time for handwashing!? Handwashing versus alcoholic rub: can we afford $100 \%$ compliance? Infect Control Hosp Epidemiol 1997;18:205-208.

24 Larson E: Skin hygiene and infection prevention: more of the same or different approaches? Clin Infect Dis 1999;29:12871294.

25 McAteer J, Stone S, Roberts J, Michie S, Fuller C, Slade R, Charlett A, Cookson B, Cooper B, Duckworth G, Hayward A, Jeane A, Teare L: Use of performance feedback to increase healthcare worker hand-hygiene behaviour. J Hosp Infect 2007;66:291-292; author reply 292-293.

26 Cromer AL, Latham SC, Bryant KG, Hutsell S, Gansauer L, Bendyk HA, Steed R, Carney MC: Monitoring and feedback of hand hygiene compliance and the impact on facilityacquired methicillin-resistant Staphylococcus aureus. Am J Infect Control 2008;36: 672-677.

27 Bittle MJ, LaMarche S: Engaging the patient as observer to promote hand hygiene compliance in ambulatory care. Jt Comm J Qual Patient Saf 2009;35:519-525.

28 Sahud AG, Bhanot N, Radhakrishnan A, Bajwa R, Manyam H, Post JC: An electronic hand hygiene surveillance device: a pilot study exploring surrogate markers for hand hygiene compliance. Infect Control Hosp Epidemiol 2010;31:634-639.

-29 Jenner EA, Fletcher BC, Watson P, Jones FA, Miller L, Scott GM: Discrepancy between self-reported and observed hand hygiene behaviour in healthcare professionals. J Hosp Infect 2006;63:418-422.

30 Larson E: Handwashing: it is essential even when gloves are used. Servir 1990;38:275279 (in Portuguese). 